\title{
ON THE MIXING PROPERTIES OF PIECEWISE EXPANDING MAPS UNDER COMPOSITION WITH PERMUTATIONS, II: MAPS OF NON-CONSTANT ORIENTATION
}

\author{
NIGEL P. BYOTT, CONGPING LIN, AND YIWEI ZHANG
}

\begin{abstract}
For an integer $m \geq 2$, let $\mathcal{P}_{m}$ be the partition of the unit interval $I$ into $m$ equal subintervals, and let $\mathcal{F}_{m}$ be the class of piecewise linear maps on $I$ with constant slope $\pm m$ on each element of $\mathcal{P}_{m}$. We investigate the effect on mixing properties when $f \in \mathcal{F}_{m}$ is composed with the interval exchange map given by a permutation $\sigma \in S_{N}$ interchanging the $N$ subintervals of $\mathcal{P}_{N}$. This extends the work in a previous paper [N.P. Byott, M. Holland and Y. Zhang, DCDS, 33, (2013) 3365-3390], where we considered only the "stretch-and-fold" $\operatorname{map} f_{\mathrm{sf}}(x)=m x \bmod 1$.
\end{abstract}

\section{InTROdUCtion AND STATEMENT OF RESUlts}

A natural question about a dynamical system is how fast it mixes measurable subsets of its domain. In the setting of discrete time piecewise smooth onedimensional expanding maps, the rate of decay of correlations for functions of bounded variation gives a quantitative interpretation of the speed of mixing, and is governed by the isolated spectrum of the transfer operator on the space of such functions (see [3,6] and the references therein). As there is no general approach to determine this isolated spectrum explicitly, detailed studies of specific families of maps, as for example in [9] and [10], are an important step towards a better understanding of quantitative mixing phenomena.

For simplicity, we focus our discussion on piecewise linear Markov maps. A nice property of such maps is that the corresponding transfer operator has a finite matrix representation. It is perhaps surprising that, even in this special situation, the precise determination of the mixing rate is already a non-trivial task [21, 22]. On the other hand, using an Ulam-like construction [4, 23], every expanding (nonlinear) Markov map can be approximated by a sequence of piecewise linear maps. In many circumstances, we can use the mixing rate of high order piecewise linear maps to bound or approximate the mixing rate of the original non-linear maps [8, 21, 22]. (This is extended in $[14,15]$ to the settings of multi-dimensional expanding maps and of Anosov maps.) Historically, on the basis of explicit calculations for some topologically mixing piecewise linear Markov maps (namely the skew doubling maps and skew tent maps), Badii et al. [2] conjectured that, for every topologically mixing and expanding Markov map, the mixing rate can be bounded from above in terms of a (generalized) Lyapunov exponent. A counterexample was soon obtained [8] by making a non-linear perturbation to these maps. Indeed, as observed in $[10,17,21,22]$, the Lyapunov exponent and the topological entropy only provide lower bounds for the mixing rate via bounded variation observations.

Date: June 10, 2015. 
In a continuous time setting, a novel aspect of mixing was considered in [1]. The domain of a one-dimensional diffusion process was divided into equal subintervals, and the underlying function was composed with an interval exchange map corresponding to a permutation of these. This typically results in faster mixing than the diffusion alone.

In view of these results, it is of interest to investigate the analogous effect of composition with a permutation for amenable examples of piecewise linear Markov maps. Such an investigation began in our previous paper [7]. In particular, we considered the "stretch-and-fold" map $f_{\text {sf }}(x)=m x \bmod 1$ for an integer $m \geq 2$ on $I=[0,1]$. (When $m=2$, this is the well-known "doubling map"). When $I$ is divided into $N \geq m$ equal subintervals, and $f$ is composed with a permutation $\sigma$ of these, the Lyapunov exponent and topological entropy are unchanged, but, in contrast to the results of [1], mixing typically becomes slower. Indeed, there may be permutations $\sigma$ such that the new map $\sigma \circ f_{\text {sf }}$ fails to be topologically mixing. Under the hypothesis $\operatorname{gcd}(m, N)=1$, we showed that $\sigma \circ f_{\text {sf }}$ is topologically mixing for all permutations $\sigma$, and we determined explicitly the worst mixing attained as $\sigma$ varies. In fact, the mixing can be made arbitrarily slow by allowing $N$ to increase. This not only shows a striking contrast between the effect of composing with a permutation in the continuous and discrete time settings, but also adds to the collection of piecewise expanding interval maps where mixing rates can be explicitly determined. Indeed, these maps provide an alternative counterexample to the conjecture of Badii et al., in which no non-linear perturbations are required: the Markov structure is changed in an essentially combinatorial manner, and only piecewise linear maps are used. Moreover, since we consider Markov partitions with equal subintervals, this has the advantage that the mixing rate can be calculated exactly rather than approximated numerically.

In the present paper, we continue this investigation by regarding $f_{\text {sf }}$ as the simplest member of a family $\mathcal{F}_{m}$ of interval maps with piecewise constant integer slope $\pm m$. More precisely, for fixed $m \geq 2$, and for any sequence $\epsilon_{1}, \ldots, \epsilon_{m} \in$ $\{-1,1\}^{m}$, we take $f_{\epsilon_{1}, \ldots, \epsilon_{m}}$ to be the interval map with slope $m \epsilon_{j}$ on the interval $((j-1) / m, j / m)$. Explicitly,

$$
f_{\epsilon_{1}, \ldots, \epsilon_{m}}(x)= \begin{cases}m x-j+1 & \text { if } j-1 \leq m x<j \text { with } \epsilon_{j}=1 \\ j-m x & \text { if } j-1 \leq m x<j \text { with } \epsilon_{j}=-1\end{cases}
$$

We consider the family of $2^{m}$ maps $\mathcal{F}_{m}=\left\{f_{\epsilon_{1}, \ldots, \epsilon_{m}}: \epsilon_{j}= \pm 1\right\}$. Taking $\epsilon_{j}=1$ for all $j$ gives the stretch-and-fold map $f_{\text {sf }}$ with piecewise constant slope $m$. At the other extreme, taking $\epsilon_{j}=(-1)^{j-1}$ gives a map with $m-1$ changes of slope. We shall refer to this as the $m$-fold zigzag map, writing $f_{\mathrm{zz}}$ for $f_{1,-1,1, \ldots}$. (In the case $m=2$, the zigzag map $f_{1,-1}$ is just the familiar "tent map" $f_{\text {tent }}$.) We could equally well take the inverted zigzag map with $\epsilon_{j}=(-1)^{j}$, and we write $f_{\text {izz }}$ for $f_{-1,+1,-1, \ldots}$.

Let $\mathcal{P}_{N}$ be the partition of $I$ into $N \geq m$ equal subintervals. We write $S_{N}$ for the symmetric group, consisting of all $N$ ! permutations $\sigma$ of $\{1,2, \ldots, N\}$. Abusing notation, we also write $\sigma$ for the interval exchange map given by the corresponding permutation of the subintervals of $\mathcal{P}_{N}$ :

$$
\sigma(x)=x+(\sigma(j)-j) / N \text { if }(j-1) / N \leq x<j / N
$$


For each $f \in \mathcal{F}_{m}$ and each $\sigma \in S_{N}$, the map $g=\sigma \circ f$ has piecewise constant slope $\pm m$. It follows that $g$ has topological entropy $\log m$, and that the Lyapunov exponent of $g$ is also $\log m$; these quantities are therefore unaffected by composition with $\sigma$ and hence independent of $N$. The purpose of this paper is to investigate how the mixing rate is affected by the combinatorial operation of composition with $\sigma$. In particular, we shall determine to what extent the mixing properties of $f$ are neutralized by composition with an appropriate choice of $\sigma$.

Before describing in detail the results of this paper, we explain the notion of mixing rate we shall use. We quantify the speed of mixing in terms of the decay of correlations for observables of bounded variation. The context is briefly summarized below, and we refer the readers to [6] for more detailed information. Let $g: I \longrightarrow \mathbb{R}$ be a piecewise expanding map and suppose for the moment that $g$ is topologically mixing. Then $g$ admits a unique absolutely continuous invariant probability measure $\mu$. Let $B V$ denote the Banach space of functions of bounded variation on $I$ (modulo the Lebesgue measure) under the bounded variation norm. For $\phi, \psi \in B V$ and for $n \geq 1$, we consider the correlation function

$$
C_{\phi, \psi}(n):=\int_{I} \phi\left(g^{n}(x)\right) \psi(x) d \mu(x)-\int_{I} \phi(x) d \mu(x) \int_{I} \psi(x) d \mu(x) .
$$

Definition 1.1. Let $g$ be any piecewise expanding map on $I$. The mixing rate $\tau(g)$ of $g$ is given by

$$
\tau(g):=\inf \left\{\tau \geq 0: C_{\phi, \psi}(n):=O\left(\tau^{n}\right) \text { as } n \rightarrow \infty \text { for all } \phi, \psi \in B V\right\} .
$$

If $g$ is topologically mixing then $\tau(g)<1$ by [24, Corollary 3.10]. Conversely, if $g$ is not topologically mixing then $\tau(g)=1$, as can be seen be taking $\phi, \psi$ to be the characteristic functions on suitable subsets of $I$.

We now restrict attention to maps of the form $g=\sigma \circ f$ with $f \in \mathcal{F}_{m}$ and $\sigma \in S_{N}$. In this situation, the absolutely continuous invariant probability measure $\mu$ is always the Lebesgue measure. We will consider the worst mixing rate attained by the maps $\sigma \circ f$ as $\sigma$ varies.

Definition 1.2. Given $f \in \mathcal{F}_{m}$ and $N \geq m$, we set

$$
\tau^{(N)}(f):=\max \left\{\tau(\sigma \circ f): \sigma \in S_{N}\right\} .
$$

The main results of this paper will be expressed in terms of $\tau^{(N)}(f)$. Our first result characterizes those $f$ such that $\sigma \circ f$ is topologically mixing for all $\sigma \in S_{N}$.

Theorem 1. Let $f \in \mathcal{F}_{m}$. Then $\tau^{(N)}(f)=1$ if and only if either

(i) $m \mid N$, or

(ii) $m \mid 2 N$ and $f=f_{\mathrm{zz}}$ or $f_{\mathrm{izz}}$.

In particular, if $m \nmid 2 N$ then $\sigma \circ f$ is topologically mixing for all $f \in \mathcal{F}_{m}$ and all $\sigma \in S_{N}$.

Although the family $\mathcal{F}_{m}$ contains $2^{m}$ maps, we will see in Lemma 2.8 below that $\tau^{(N)}\left(f_{\epsilon_{1}, \ldots, \epsilon_{m}}\right)$ is unchanged both by reversing the sequence $\epsilon_{1}, \ldots, \epsilon_{m}$ and by replacing $\epsilon_{j}$ by $-\epsilon_{j}$ for all $j$. In particular $\tau^{(N)}\left(f_{\mathrm{izz}}\right)=\tau^{(N)}\left(f_{\mathrm{zz}}\right)$. Up to these symmetries, $\mathcal{F}_{2}$ contains just two maps (namely, the doubling map $f_{\text {sf }}$ and the tent map $\left.f_{\mathrm{zz}}=f_{\text {tent }}\right), \mathcal{F}_{3}$ contains three maps as illustrated in Figure $1.1, \mathcal{F}_{4}$ contains four maps, and $\mathcal{F}_{5}$ contains 10 maps. 

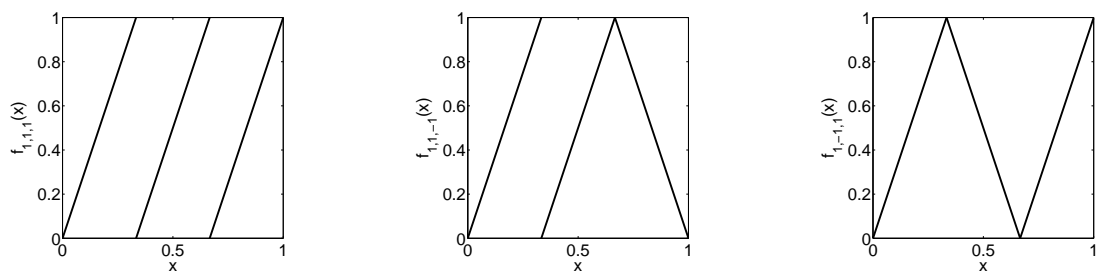

Figure 1.1. The family of maps $\mathcal{F}_{3}=\left\{f_{s f}, f_{1,1,-1}, f_{z z}\right\}$.

The maps $f_{\text {sf }}$ and $f_{\text {zz }}$ are extremal elements of $\mathcal{F}_{m}$ in that they have respectively the smallest and largest number of changes of slope. Our next result shows that, in a certain sense, no $f \in \mathcal{F}_{m}$ can have worse asymptotic mixing behavior than $f_{\mathrm{zz}}$.

Theorem 2. For each odd $m \geq 3$ there is a constant $c(m)>0$ such that the following holds. For any sequence of integers $N_{i}>m$ such that $N_{i} \rightarrow \infty$ as $i \rightarrow \infty$ and $\operatorname{gcd}\left(m, N_{i}\right)=1$ for each $i$, and for any $f \in \mathcal{F}_{m}$, we have

$$
\liminf _{i \rightarrow \infty} \frac{1-\tau^{\left(N_{i}\right)}(f)}{1-\tau^{\left(N_{i}\right)}\left(f_{\mathrm{zz}}\right)} \geq c(m) .
$$

Explicitly, we may take $c(m)=12 /\left(m^{4}-m^{2}\right)$.

In the case $m=3$, we have the more precise statement:

$$
\tau^{(N)}(f) \leq \tau^{(N)}\left(f_{\mathrm{zz}}\right) \text { for all } f \in \mathcal{F}_{3} \text { and for all } N \text { with } \operatorname{gcd}(3, N)=1 \text {. }
$$

Note that, by Theorem 1 , the condition $\operatorname{gcd}\left(m, N_{i}\right)=1$, with $m$ odd, ensures that $\tau^{\left(N_{i}\right)}(f), \tau^{\left(N_{i}\right)}\left(f_{\mathrm{zz}}\right)<1$ for all $i$.

The proof of Theorem 2 has two main ingredients. One of these is a result of Fiedler [11] which bounds the subleading eigenvalue of a doubly stochastic matrix away from 1. It is well-known that there is a strong connection between mixing and spectral gaps for the transfer operator, but we are not aware of any previous work where a general bound on the eigenvalues of a stochastic matrix has been used to obtain explicitly quantitative information on the limiting behavior of mixing rates. The other ingredient is the following exact result on the zigzag map.

Theorem 3. Let $N \geq m \geq 2$, and let $d=\operatorname{gcd}(m, 2 N)$. Then

$$
\tau^{(N)}\left(f_{\mathrm{zz}}\right)=\frac{d \sin (m \pi / 2 N)}{m \sin (d \pi / 2 N)} .
$$

In particular, if $\operatorname{gcd}(m, 2 N)=1$ then

$$
\tau^{(N)}\left(f_{\mathrm{zz}}\right)=\frac{\sin (m \pi / 2 N)}{m \sin (\pi / 2 N)} .
$$

The techniques used to prove Theorem 3 are extensions of those used for the stretch-and-fold map in [7], and also allow us to give the following improvement of [7, Theorem 2.2(ii)] (where $m$ and $N$ were assumed coprime). 
Theorem 4. Let $N \geq m \geq 2$ and let $d=\operatorname{gcd}(m, N)$. Then

$$
\tau^{(N)}\left(f_{\mathrm{sf}}\right)=\frac{d \sin (m \pi / N)}{m \sin (d \pi / N)}
$$

A case of particular interest is $f_{\mathrm{zz}}$ for $m=2$, which is the much-studied tent map. As $\operatorname{gcd}(2,2 N)=2$, Theorem 3 merely tells us that $\tau^{(N)}\left(f_{\text {tent }}\right)=1$ for all $N$ : there are some permutations $\sigma \in S_{N}$ such that $\sigma \circ f$ is not topologically mixing. Note, however, that iterates of $f_{\text {tent }}$ do not share this property; the second iterate $f_{\text {tent }}^{2}$ of $f_{\text {tent }}$ is just the zigzag map for $m=4$, for which Theorem 3 gives $\tau^{(N)}\left(f_{\mathrm{zz}}\right)<1$ for all odd $N \geq 5$.

In the cases where $\tau^{(N)}(f)=1$, it is natural to exclude those permutations $\sigma$ where $\sigma \circ f$ is not topologically mixing. We therefore give a modified version of Definition 1.2:

\section{Definition 1.3.}

$$
\tau_{\text {mix }}^{(N)}(f):=\max \left\{\tau(\sigma \circ f): \sigma \in S_{N} \text { with } \sigma \circ f \text { topologically mixing }\right\} .
$$

When $\sigma \circ f$ fails to be topologically mixing for some $\sigma$, we have $\tau_{\text {mix }}^{(N)}(f)<$ $\tau^{(N)}(f)=1$ and $\tau_{\text {mix }}^{(N)}(f)$ is obtained by maximizing over a proper subset of $S_{N}$. Although we have no general method for approaching maximization problems of this type, we are able to give a partial result in the case of the tent map $f_{\text {tent }}=f_{\mathrm{zz}}$ for $m=2$. We give a lower bound for the worst mixing rate $\tau_{\text {mix }}^{(N)}\left(f_{\text {tent }}\right)$. As explained in $\S 2$, the mixing rate of $g=\sigma \circ f$ can be obtained as the subleading eigenvalue of the transfer operator $\mathcal{L}_{g}$ of $g$. We are able to obtain some geometric restrictions on the location in the complex plane of the nonleading eigenvalues of $\mathcal{L}_{g}$ for $g=\sigma \circ f_{\text {tent }}$.

Theorem 5. Let $m=2$ and $N>2$ be odd. Then $\tau_{\text {mix }}^{(N)}\left(f_{\text {tent }}\right) \geq \cos (\pi / N)$. Moreover, if $g=\sigma \circ f_{\text {tent }}$ for some $\sigma \in S_{N}$, and $g$ is topologically mixing, then any nonleading eigenvalue $\lambda$ of $\mathcal{L}_{g}$ lies in the compact convex region of $\mathbb{C}$ given by the two inequalities

$$
-\cos ^{2}\left(\frac{\pi}{2 N}\right) \leq \operatorname{Re}(\lambda) \leq \cos \left(\frac{\pi}{N}\right)
$$

and

$$
\operatorname{Re}(\lambda)+|\operatorname{Im}(\lambda)| \tan \left(\frac{\pi}{N}\right) \leq 1
$$

Some examples of the regions given in Theorem 5 are illustrated by Figure 1.2.

Numerical calculations suggest that the lower bound on $\tau_{\text {mix }}^{(N)}\left(f_{\text {tent }}\right)$ in Theorem 5 is sharp, so that in fact $\tau_{\text {mix }}^{(N)}\left(f_{\text {tent }}\right)=\cos (\pi / N)$.

\section{Outline of the paper:}

In $\S 2$, we show how $\tau^{(N)}(f)$ can be calculated in terms of Markov matrices for $f \in \mathcal{F}_{m}$. We then turn in $\S 3$ to the exact values of $\tau^{(N)}(f)$ for the special cases $f=f_{\mathrm{zz}}$ and $f_{\mathrm{sf}}$, giving the proofs of Theorems 3 and 4 . We also prove the characterization of those $f$ with $\tau^{(N)}(f)=1$ in Theorem 1. Finally, Theorems 2 and 5 are proved in $\S 4$. We shall need a number of auxiliary results which involve only linear algebra. To clarify the exposition, we postpone their proofs to the Appendix $(\S 5)$. 

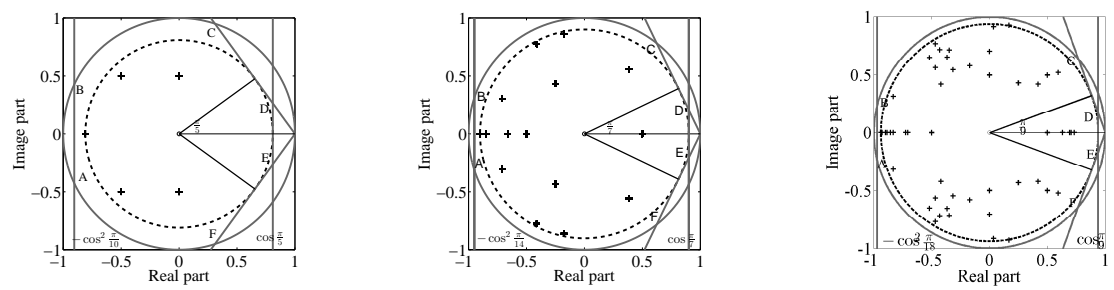

Figure 1.2. Location in the complex plane of the nonleading eigenvalues $\lambda$ of $\mathcal{L}_{\sigma \circ f_{\text {tent }}}$ for $N=5,7,9$. Each such $\lambda$ is located in the convex subset which is bounded by the line segments $A B, C D, D E$, $E F$ and the arcs $\overparen{\mathrm{BC}}, \overparen{\mathrm{FA}}$. The line segments $C D, D E, E F$ are tangent to the disc centered at the origin with radius $\cos \left(\frac{\pi}{N}\right)$.

\section{Mixing RAtes And MARKov MATrices}

In this section, we explain how mixing rates are related to the transfer operator and can be calculated from certain matrices. We restrict our discussion to maps of the form $g=\sigma \circ f$ with $f \in \mathcal{F}_{m}, \sigma \in S_{N}$ and $N \geq m$, since these are the only maps considered in this paper.

The transfer operator $\mathcal{L}_{g}: B V \rightarrow B V$ for $g$ is defined by

$$
\left\{\mathcal{L}_{g} \phi\right\}(x):=\sum_{g(y)=x} \frac{\phi(y)}{\left|g^{\prime}\right|(y)}, \quad \forall \phi \in B V .
$$

The essential spectral radius of $\mathcal{L}_{g}$ is

$$
r_{\text {ess }}(g):=\inf \left\{r \geq 0: \lambda \in \operatorname{Spec}\left(\mathcal{L}_{g}\right),|\lambda|>r \Longrightarrow \lambda \text { is isolated }\right\},
$$

where the spectrum is taken on the space $B V$. As $g$ has piecewise constant slope $\pm m$, Keller's formula [18, Theorem 1] gives $r_{\text {ess }}(g)=1 / m$. The isolated eigenvalues $\lambda$ with $|\lambda|>r_{\text {ess }}(g)$ are of finite multiplicity and satisfy $|\lambda| \leq 1$. The eigenvalue 1 always occurs. Moreover, $g$ is topologically mixing if and only if the eigenspace for the eigenvalue 1 has dimension 1 and there are no other eigenvalues $\lambda$ with $|\lambda|=1$. In this case, $\sup \left\{|\lambda|: \lambda \in \operatorname{Spec}\left(\mathcal{L}_{g}\right) \backslash\{1\}\right\}=\tau(g)<1$, so the transfer operator has a spectral gap.

For $k \geq 1$, let $\mathcal{P}_{k}$ denote the partition of the unit interval into the $k$ equal subintervals $I_{j}=[(j-1) / k, j / k]$ for $1 \leq j \leq k$. Each of our maps $g=\sigma \circ f$ is a Markov map with respect to $\mathcal{P}_{N m}$ with constant slope $\pm m$ on each subinterval. Let $B(g, N)=\left(b_{i j}\right)$ be the usual $\{0,1\}$ Markov transition matrix for $g$ on $\mathcal{P}_{N m}$, so $b_{i j}=1$ if and only if $I_{j}^{\circ} \subset g\left(I_{i}\right)$. We note that

$$
b_{i(h-1) m+k}=b_{i(j-1) m+1} \text { for } 1 \leq i \leq N m, 1 \leq j \leq N, 1 \leq k \leq m,
$$

since each subinterval of the partition $\mathcal{P}_{N m}$ is mapped by $g$ onto $m$ consecutive subintervals. The doubly stochastic matrix $m^{-1} B(g, N)$ can be interpreted as the 
probability transition matrix associated with $g$. This matrix is the Fredholm matrix for $g$, as discussed in $[7, \S 4.1]$, from where we have the following result.

Lemma 2.1. (i) The dynamical system given by $g$ is ergodic if and only if $B(g, N)$ is irreducible, and is topologically mixing if and only if $B(g, N)$ is primitive.

(ii) If $\lambda \in \mathbb{C}$ and $|\lambda|>r_{\text {ess }}(g)$ then $\lambda \in \operatorname{Spec}\left(\mathcal{L}_{g}\right)$ if and only if $\lambda$ is an eigenvalue of $m^{-1} B(g, N)$.

We recall that a nonnegative matrix $M$ is said to be irreducible (respectively, primitive) if, for all $i, j$, there is some $k \geq 1$ with $m_{i j}^{(k)}>0$ (respectively, there is some $k \geq 1$ with $m_{i j}^{(k)}>0$ for all $i, j$ ), where $M^{k}=\left(m_{i j}^{(k)}\right)$ for $k \geq 1$.

Let $M$ be any nonnegative matrix with constant row and column sums $c>0$. By the Frobenius-Perron theorem, every eigenvalue $\lambda$ of $M$ satisfies $|\lambda| \leq c$. The leading eigenvalue of $M$ is the eigenvalue $\lambda=c$, corresponding to the eigenvector $(1, \ldots, 1)^{T}$. Moreover, the $(N-1)$-dimensional space $\mathbb{C}_{0}^{N}=\left\{\left(x_{1}, \ldots, x_{N}\right)^{T} \in\right.$ $\left.\mathbb{C}^{N}: \sum_{j} x_{j}=0\right\}$ is stable under $M$. We shall refer to eigenvalues of $M$ on $\mathbb{C}_{0}^{N}$ as nonleading eigenvalues. Note that for a nonleading eigenvalue $\lambda$ we may have $\lambda=c$ (if $c$ is an eigenvalue of $M$ of algebraic multiplicity $>1$ ), and we may have $|\lambda|=c \neq \lambda$. It is well-known that every nonleading eigenvalue $\lambda$ of a doubly stochastic matrix $M$ satisfies $\lambda \neq 1$ (respectively, $|\lambda|<1$ ) if and only if $M$ is irreducible (respectively, primitive).

It will be convenient to define an analogue of the mixing rate $\tau(g)$ for matrices.

Definition 2.2. Let $M$ be a nonnegative $N \times N$ matrix with constant row and column sums. If $N \geq 2$, we define

$$
\tau(M)=\max \{|\lambda|: \lambda \text { is a nonleading eigenvalue of } M\},
$$

that is, $\tau(M)$ is the modulus of the subleading eigenvalue of $M$.

In the degenerate case $N=1$, we define $\tau(M)=0$.

We now explain how $\tau(g)$ can be obtained from an $N \times N$ matrix in place of the $N m \times N m$ matrix $B(g, N)$. Note that $f$ is, by definition, a Markov map on the partition $\mathcal{P}_{m}$, but not in general on the partition $\mathcal{P}_{N}$. Thus $f$, and hence also $g$, may change slope (from $m$ to $-m$ or vice versa) on a subinterval of $\mathcal{P}_{N}$.

Definition 2.3. The reduced Markov matrix $A(g, N)$ is the $N \times N$ matrix $\left(a_{i j}\right)$ given by

$$
a_{i j}=\sum_{h=1}^{m} b_{(i-1) m+h(j-1) m+1} \in\{0,1,2\} .
$$

Lemma 2.4. Let $g=\sigma \circ f$ with $f \in \mathcal{F}_{m}$ and $\sigma \in S_{N}$. Then

$$
\tau(B(g, N))=\tau(A(g, N)) .
$$

The proof of Lemma 2.4 is given in the Appendix.

Since $\tau(g) \geq r_{\text {ess }}(g)=1 / m$, the following result is then immediate from Definition 1.1, Lemma 2.1 and Lemma 2.4:

Corollary 2.5.

$$
\tau(g)=\frac{1}{m} \max \{1, \tau(A(g, N))\}
$$


The mixing rates for the maps $f \in \mathcal{F}_{m}$ themselves (with no permutation) are easily determined. In this case, $f$ is a Markov map with respect to the partition $\mathcal{P}_{m}$ since each subinterval of length $1 / m$ is mapped homeomorphically to the whole of $[0,1]$ (with either positive or negative orientation). We may therefore apply Lemma 2.1 to the Fredholm matrix $F$ for this partition (effectively taking $N=1$ ). But all the entries of this $m \times m$ matrix are $1 / m$, so its only nonleading eigenvalue is 0 , occurring with multiplicity $m-1$. Thus $\tau(F)=0$, and we have

$$
\tau(f)=1 / m \text { for each } f \in \mathcal{F}_{m} .
$$

We next make explicit the relationship between the matrices $A(g, N)$ and $A(f, N)$ (respectively, $B(g, N)$ and $B(f, N))$ where $g=\sigma \circ f$ with $\sigma \in S_{N}$. Let $P(\sigma)=\left(p_{i j}\right)$ be the $N \times N$ permutation matrix for $\sigma$ :

$$
p_{i j}=\left\{\begin{array}{lc}
1 & \text { if } j=\sigma(i), \\
0 & \text { otherwise }
\end{array}\right.
$$

and let $Q(\sigma)$ be the $N m \times N m$ permutation matrix obtained by replacing each entry 1 (respectively, 0 ) in $P(\sigma)$ by an $m \times m$ identity (respectively, zero) matrix. Then

$$
A(g, N)=A(f, N) P(\sigma), \quad B(g, N)=B(f, N) Q(\sigma) .
$$

It will be convenient to define an analogue for matrices of the quantity $\tau^{(N)}(f)$ in Definition 1.2.

Definition 2.6. Let $M$ be a nonnegative $N \times N$ matrix with constant row and column sums. Then

$$
\tau_{\text {perm }}(M)=\max \left\{\tau(M P(\sigma)): \sigma \in S_{N}\right\} .
$$

For any $\sigma \in S_{N}$, we have

$$
\tau_{\text {perm }}(M P(\sigma))=\tau_{\text {perm }}(M)=\tau_{\text {perm }}(P(\sigma) M) ;
$$

the first inequality is immediate from the definition of $\tau_{\text {perm }}$, and the second holds since $\mathbb{C}_{0}^{N}$ is stable under $P(\sigma)$ and the conjugate matrices $M P(\sigma)$ and $P(\sigma) M$ have the same eigenvalues.

It then follows from Definition 1.2, Corollary 2.5 and (2.5) that

$$
\tau^{(N)}(f)=\frac{1}{m} \max \left\{1, \tau_{\text {perm }}(A(f, N))\right\} \text { for all } f \in F_{m} .
$$

Lemma 2.7. Let $A$ be a nonnegative matrix with constant row and column sums. Then $\tau\left(A A^{T}\right)=\tau\left(A^{T} A\right) \geq \tau_{\text {perm }}(A)^{2}$. If $A$ is symmetric then $\tau_{\text {perm }}(A)=\tau(A)$.

The proof of Lemma 2.7 is given in the Appendix. The second assertion is essentially [7, Lemma 4.3].

We end this section by justifying the claim made in $\S 1$ that $\tau^{(N)}(f)$ is unchanged by certain symmetries on $\mathcal{F}_{m}$.

Lemma 2.8. Let $\left(\epsilon_{1}, \ldots, \epsilon_{m}\right) \in\{ \pm 1\}^{m}$, and let

$$
f=f_{\epsilon_{1}, \ldots, \epsilon_{m}}, \quad f_{1}=f_{-\epsilon_{1}, \ldots,-\epsilon_{m}}, f_{2}=f_{\epsilon_{m}, \ldots, \epsilon_{1}} .
$$

Then $\tau^{(N)}\left(f_{1}\right)=\tau^{(N)}\left(f_{2}\right)=\tau^{(N)}(f)$. 
Proof. The matrix $A\left(f_{1}, N\right)$ is obtained by reversing the order of the columns of $A(f, N)$, since each subinterval of length $1 / N$ is traversed in the opposite direction. Thus $A\left(f_{1}, N\right)=A\left(f_{1}, N\right) P(\tau)$, where $\tau \in S_{N}$ is the permutation given by $\tau(j)=N+1-j$ for $1 \leq j \leq N$. Similarly, $A\left(f_{2}, N\right)=P(\tau) A(f, N)$. Hence by $(2.6)$,

$$
\tau_{\text {perm }}\left(A\left(f_{1}, N\right)\right)=\tau_{\text {perm }}\left(A\left(f_{2}, N\right)\right)=\tau_{\text {perm }}(A(f, N)) .
$$

and the result follows from $(2.7)$.

\section{EXACT RESULTS}

In this section, we prove Theorems 3,4 and 1.

\subsection{Preliminary results.}

3.1.1. Eigenvectors of block matrices. Let $n \geq 1$ be a factor of $N$, say $N=d n$. We will relate $n \times n$ matrices to certain $N \times N$ matrices, partitioned into $d \times d$ blocks.

Definition 3.1. Let $A=\left(a_{i j}\right)$ be an $n \times n$ matrix. Then $A^{\uparrow}$ denotes the $d n \times d n$ matrix obtained by replacing each entry $a_{i j}$ of $A$ by a $d \times d$ matrix, each of whose entries is $a_{i j}$.

Lemma 3.2. Let $A$ be a nonnegative $d \times d$ matrix with constant row and column sums. Then $\tau\left(A^{\uparrow}\right)=d \tau(A)$.

The proof of Lemma 3.2 is given in the Appendix.

3.1.2. Matrices with a double symmetry property. Let $J=J_{N}$ denote the "backwards identity" matrix $\left(\delta_{i, N+1-j}\right)_{1 \leq i, j \leq N}$, where $\delta_{i j}$ is the Kronecker delta. Then $J M$ (respectively $M J$ ) is the matrix obtained from an $N \times N$ matrix $M$ by reversing the order of its rows (respectively, columns). We also write $\widetilde{J}$ for the $2 N \times 2 N$ matrix $J_{2 N}$ of the same form.

We will require the following result, whose (easy) proof we leave to the reader.

Lemma 3.3. Let $\widetilde{A}$ be a $2 N \times 2 N$ matrix with constant column sums such that $\widetilde{J} \widetilde{A}=\widetilde{A}=\widetilde{A} \widetilde{J}$. Then

$$
\widetilde{A}=\left(\begin{array}{cc}
A & A J \\
J A & J A J
\end{array}\right)
$$

for some $N \times N$ matrix A with constant column sums. Moreover,

$$
\tau(\widetilde{A})=2 \tau(A) .
$$

3.1.3. Circulant matrices. Let $m, N$ be natural numbers such that $1 \leq m \leq N$ and $\operatorname{gcd}(m, N)=1$. We define

$$
\delta= \begin{cases}(1-m) / 2 & \text { if } m \text { is odd; } \\ (1-m+N) / 2 & \text { if } m \text { is even; }\end{cases}
$$

and set $C=C(m, N)=\left(c_{i j}\right)_{1 \leq i, j \leq N}$ with

$$
c_{i j}= \begin{cases}1 & \text { if } j \equiv i+\delta+r \bmod N \text { with } 0 \leq r<m \\ 0 & \text { otherwise }\end{cases}
$$


Then $C$ is a symmetric circulant matrix. By $[7,(24)$ and Proposition 6], we have

$$
\tau(C)=\frac{\sin (m \pi / N)}{\sin (\pi / N)} .
$$

Proposition 3.4. Let $D=C+C J_{N}$. Then

$$
\tau(D)=\frac{2 \sin (m \pi / N)}{\sin (\pi / N)}
$$

The proof of Proposition 3.4 is given in the Appendix.

3.2. Worst mixing rate for $f_{\mathrm{sf}}$ and $f_{\mathrm{zz}}$. In this subsection, we prove Theorems 3 and 4 , giving the worst mixing rate in the special cases of the zigzag map $f_{\mathrm{zz}}$ and the stretch-and-fold map $f_{\text {sf }}$. We begin with the easier case $f_{\text {sf }}$.

Proof of Theorem 4. Let $d=\operatorname{gcd}(m, N)$, and write $h=m / d$ and $n=N / d$. Let $A=A\left(f_{\mathrm{sf}}, N\right)$. Then $A$ contains $n$ distinct rows, each occurring $d$ times. Let $C_{0}$ denote the symmetric circulant matrix $C(h, n)$ as in $\S 3.1 .3$, which is defined since $\operatorname{gcd}(h, n)=1$, and let $C=C_{0}^{\uparrow}$ be the corresponding $N \times N$ block matrix as given by Definition 3.1. Then $A$ and $C$ have the same rows, so $C=P(\sigma) A$ for some permutation $\sigma \in S_{N}$. Hence $\tau_{\text {perm }}(A)=\tau_{\text {perm }}(C)$. Since $C$ is symmetric, $\tau_{\text {perm }}(C)=\tau(C)$ by Lemma 2.7. But by Lemma 3.2 and (3.2), we have

$$
\tau(C)=d \tau\left(C_{0}\right)=\frac{d \sin (h \pi / n)}{\sin (\pi / n)}=\frac{d \sin (m \pi / N)}{\sin (d \pi / N)} .
$$

Then (2.7) gives

$$
\tau^{(N)}\left(f_{\mathrm{sf}}\right)=\frac{1}{m} \tau_{\text {perm }}(A)=\frac{d \sin (m \pi / N)}{m \sin (d \pi / N)} .
$$

We now turn to the $m$-fold zigzag map $f_{\mathrm{zz}}$.

Proof of Theorem 3. We will relate the matrices $A=A\left(f_{\mathrm{zz}}, N\right)$ and $\widetilde{A}=A\left(f_{\mathrm{sf}}, 2 N\right)$. Explicitly, we have $\widetilde{A}=\left(\tilde{a}_{i j}\right)_{1 \leq i, j \leq 2 N}$, where

$$
\tilde{a}_{i j}= \begin{cases}1 & \text { if } j \equiv m i-r \bmod 2 N \text { with } 0 \leq r<m ; \\ 0 & \text { otherwise. }\end{cases}
$$

Let $J=J_{N}$ and $\widetilde{J}=J_{2 N}$ as before. It is routine to verify that $\widetilde{A}$ commutes with $\widetilde{J}$. Write $E=\widetilde{A}+\widetilde{A} \widetilde{J}$. We have $\widetilde{J} E=E=E \widetilde{J}$. The upper left-hand quarter of the $2 N \times 2 N$ matrix $E$ is precisely the $N \times N$ matrix $A\left(f_{\mathrm{zz}}, N\right)$. (This reflects the fact that we may regard $f$ as giving either a dynamical system with states $1, \ldots$, $N$ corresponding to the subintervals of the partition $\mathcal{P}_{N}$, or a system with states $1^{+}, \ldots N^{+}, N^{-}, \ldots, 1^{-}$; the states $j^{+}, j^{-}$correspond to the subinterval $I_{j}$ with an orientation depending on the slope of $f$. The construction of $E$ recombines states $j^{+}, j^{-}$into the single state $j$.) By Lemma 3.3, we then have

$$
E=\left(\begin{array}{cc}
A & A J \\
J A & J A J
\end{array}\right)
$$

Now consider the effect of replacing $f_{\mathrm{zz}}$ by $g=\sigma \circ f_{\mathrm{zz}}$ for some $\sigma \in S_{N}$. This replaces $A$ by $A P(\sigma)$, where $P(\sigma)$ is the permutation matrix corresponding to $\sigma$, 
and so replaces $\widetilde{A}$ by $\widetilde{A} \tilde{P}(\sigma)$, where $\tilde{P}(\sigma)$ is the $2 N \times 2 N$ permutation matrix

$$
\tilde{P}(\sigma)=\left(\begin{array}{cc}
P(\sigma) & 0 \\
0 & J P(\sigma) J
\end{array}\right) .
$$

Thus $E$ is replaced by the matrix

$$
\left(\begin{array}{cc}
A P(\sigma) & A P(\sigma) J \\
J A P(\sigma) & J A P(\sigma) J
\end{array}\right)=E \tilde{P}(\sigma) .
$$

Using Lemma 3.3 again, we have

$$
2 \tau(A P(\sigma))=\tau(E \tilde{P}(\sigma)) .
$$

Next, let $d=\operatorname{gcd}(m, 2 N)$, and write $h=m / d$ and $n=2 N / d$. Let $C_{0}=C(h, n)$, and let $C=C_{0}^{\uparrow}$. Then $C=\widehat{P}(\pi) A\left(f_{\text {sf }}, 2 N\right)$ where $\widehat{P}(\pi)$ is the $2 N \times 2 N$ permutation matrix for some $\pi \in S_{2 N}$, as in (2.4). Set $D=C+C \widetilde{J}$ and $D_{0}=C_{0}+C_{0} J_{n}$, so that $D=D_{0}^{\uparrow}$, and let $\tilde{P}(\phi)$ be defined as in (3.4) with $\phi \in S_{N}$ given by

$$
\phi(i)= \begin{cases}\pi(i) & \text { if } \pi(i) \leq N \\ 2 N+1-\pi(i) & \text { if } \pi(i)>N,\end{cases}
$$

Then we have

$$
\widehat{P}(\pi)+\widehat{P}(\pi) \widetilde{J}=\tilde{P}(\phi)+\tilde{P}(\phi) \widetilde{J} .
$$

Since $\widetilde{A}$ commutes with $\widetilde{J}$, it follows that $D=\tilde{P}(\phi) E$. For each $\sigma \in S_{N}$, we therefore have

$$
2 \tau(A P(\sigma))=\tau\left(\tilde{P}(\phi)^{-1} D \tilde{P}(\sigma)\right)=\tau(D \tilde{P}(\sigma \phi)) .
$$

Each matrix $\tilde{P}(\sigma \phi)$ is a permutation matrix $\widehat{P}(\pi)$ for some $\pi \in S_{2 N}$, but not every $\pi \in S_{2 N}$ can occur. Taking the maximum over $\sigma \in S_{N}$, we have

$$
2 \tau_{\text {perm }}(A)=\max _{\sigma \in S_{N}} \tau(D \tilde{P}(\sigma \phi)) \leq \max _{\pi \in S_{2 N}} \tau(D \widehat{P}(\pi))=\tau_{\text {perm }}(D) .
$$

On the other hand, taking $\sigma=\phi^{-1}$, we have

$$
2 \tau_{\text {perm }}(A) \geq \tau(D)
$$

But $D$ is a real symmetric matrix, so $\tau_{\text {perm }}(D)=\tau(D)$ by Lemma 2.7. Hence we have $2 \tau_{\text {perm }}(A)=\tau(D)$.

We now calculate $\tau(D)$. Since $D=D_{0}^{\uparrow}$, we have $\tau(D)=d \tau\left(D_{0}\right)$ by Lemma 3.2. Since $D_{0}=C(h, n)+C(h, n) J_{n}$, Proposition 3.4 tells us that

$$
\tau\left(D_{0}\right)=\frac{2 \sin (h \pi / n)}{\sin (\pi / n)}=\frac{2 \sin (m \pi / 2 N)}{\sin (d \pi / 2 N)},
$$

so that

$$
\tau(D)=\frac{2 d \sin (m \pi / 2 N)}{\sin (d \pi / 2 N)} .
$$

Finally, from (2.7), we have

$$
\tau^{(N)}\left(f_{\mathrm{zZ}}\right)=\frac{1}{m} \tau_{\text {perm }}(A)=\frac{1}{2 m} \tau(D)=\frac{d \sin (m \pi / 2 N)}{m \sin (d \pi / 2 N)} .
$$

This completes the proof of Theorem 3 . 
3.3. When the mixing rate is 1 . In this subsection, we prove Theorem 1. To do so, we will use the facts that $\tau^{(N)}\left(f_{\mathrm{sf}}\right)=1$ if $m \mid N$ and that $\tau^{(N)}\left(f_{\mathrm{zZ}}\right)=1$ if $m \mid 2 N$. These follow from Theorem 4 (or [7, Theorem 2.1(ii)]) and from Theorem 3 respectively, but could easily be verified directly.

Let $A=\left(a_{i j}\right)$ be any nonnegative matrix with constant row and column sums $m>0$. We introduce a corresponding "row relation" $R$ on the indexing set $\mathcal{I}=\{1, \ldots, N\}$ by writing $i R j$ if and only if there is some $h \in \mathcal{I}$ with $a_{h i} a_{h j} \neq 0$. Thus $i R j$ if and only if there is some state $h$ from which both $i$ and $j$ can be reached in one step. The relation $R$ is reflexive and symmetric, but not necessarily transitive. We write $\approx$ for the transitive closure of $R$. Then $\approx$ is an equivalence relation on $\mathcal{I}$. Since both the row and column sums of $A$ are constant, it follows that if $a_{h i} \neq 0$, then there is a path from $i$ back to $h$. Thus if $i R j$ then $i$ and $j$ are in the same irreducible component of $\mathcal{I}$ for $A$. (The converse need not to be true; for example, when $m=2$ the matrix $A\left(f_{\mathrm{zz}}, 3\right)$ has 2 equivalence classes under $\approx$ but only one irreducible component.)

We now apply this to our reduced Markov matrices $A(f, N)$.

Lemma 3.5. Let $f \in \mathcal{F}_{m}$ and $N \geq m$, and suppose that neither of the conditions (i) $m \mid N$, or

(ii) $m \mid 2 N$ and $f=f_{\mathrm{zz}}$ or $f_{\mathrm{izz}}$.

from Theorem 1 hold. Then $A(f, N)$ is irreducible, and $A(f, N)^{T} A(f, N)$ is primitive.

Proof. Let $f=f_{\epsilon_{1}, \ldots, \epsilon_{m}}$ with $\epsilon_{1}, \ldots, \epsilon_{m}= \pm 1$, let $A=\left(a_{i j}\right)$ be the matrix $A(f, N)$, and let $\approx$ be the equivalence relation corresponding to $A$ as above. To show that $A$ is irreducible, we will verify that $\mathcal{I}$ consists of a single equivalence class under $\approx$.

We suppose that $\epsilon_{1}=+1$, the case $\epsilon_{1}=-1$ being similar.

As (i) does not hold, we may write $N=q m+r$ with $0<r<m$. Now $f$ has slope $+m$ on $(0,1 / m)$, so we have $a_{i, i m-m+1}=a_{i, i m-m+2}=\cdots=a_{i, i m}=1$ for $1 \leq i \leq q$, and $a_{q+1, q m+1}, \ldots, a_{q+1, N}>0$. Thus the first $q$ rows of $A$ yield

$$
i m-m+1 \approx i m-m+2 \approx \cdots \approx i m \text { for } 1 \leq i \leq q,
$$

and the next row yields

$$
q m+1 \approx q m+2 \approx \ldots \approx N .
$$

This shows that there are at most $q+1$ equivalence classes under $\approx$.

Now since neither (i) nor (ii) holds, some row of $A$ starts with a block of 0 's of length $<m$. Thus, for some $j$, we have $a_{j m} a_{j m+1} \neq 0$. Then, for $2 \leq i \leq m$, we have $a_{k i m} a_{k-1} i m+1>0$ with $k=j+i-1$ or $j-i+1$. Hence $i m \approx i m+1$ for $1 \leq i \leq m$. Together with (3.5) and (3.6), this shows that there is a single equivalence class under $\approx$. Hence $A$ is irreducible.

We now consider the matrix $B=A^{T} A$, which has constant row and column sums $m^{2}$. Let us write $R_{A}, R_{B}$ for the row relations $R$ corresponding to $A, B$ respectively, and similarly for $\approx_{A}, \approx_{B}$. If $i R_{A} j$ then there is some $h$ with $a_{h i}$, $a_{h j}>0$. As the column sums of $A^{T}$ are $m>0$, there is some $g \in \mathcal{I}$ such that $a_{g h}^{T}>0$. We then have $b_{g i}, b_{g j}>0$, so $i \approx_{B} j$. As there is a single equivalence class under $\approx_{A}$, the same must be true for $\approx_{B}$. Thus $B$ is irreducible. This means that every nonleading eigenvalue $\lambda$ of $B$ satisfies $\lambda \neq m^{2}$. But $B$ is a positive semidefinite symmetric matrix, so its eigenvalues are real and nonnegative. Hence 
$|\lambda| \neq m^{2}$, showing that $B$ is primitive.

Proof of Theorem 1. If $\tau^{(N)}(f)=1$ then by (2.7) there is some $\sigma \in S_{N}$ such that the matrix $A(\sigma \circ f, N)=A(f, N) P(\sigma)$ is not irreducible. Then $A(\sigma \circ f, N)$ has subleading eigenvalue $\lambda$ with $|\lambda|=m$, so that $A(\sigma \circ f, N)^{T} A(\sigma \circ f, N)=$ $P(\sigma)^{-1} A(f, N)^{T} A(f, N) P(\sigma)$ has subleading eigenvalue $m^{2}$, and hence so does its conjugate $A(f, N)^{T} A(f, N)$. Thus $A(f, N)^{T} A(f, N)$ is not primitive, and by Lemma 3.5, either (i) or (ii) must hold. This proves the "only if" direction of Theorem 1.

We now prove the converse. First, suppose that (i) holds, say $N=q m$. Then $\tau^{(N)}\left(f_{\text {sf }}\right)=1$ by Theorem 4 . Now for any $f \in \mathcal{F}_{m}$, the first $q$ rows of $A(f, N)$ are the same as the corresponding rows in $A\left(f_{\mathrm{sf}}, N\right)$ (possibly in reverse order), and each subsequent block of $q$ rows simply repeats the first block (possibly in reverse order). Thus $A(f, N)$ is obtained from $A\left(f_{\text {sf }}, N\right)$ by some permutation of the rows, so that $\tau^{(N)}(f)=\tau^{(N)}\left(f_{\text {sf }}\right)$. Hence $\tau^{(N)}(f)=1$ for all $f \in \mathcal{F}_{m}$. Finally, suppose that (ii) holds. Then $\tau^{(N)}\left(f_{\mathrm{zz}}\right)=1$ by Theorem 3 , and $\tau^{(N)}\left(f_{\mathrm{izz}}\right)=\tau^{(N)}\left(f_{\mathrm{zz}}\right)$ by Lemma 2.8 .

\section{Asymptotic Results}

In this section we prove Theorems 2 and 5 .

4.1. Eigenvalues of doubly stochastic matrices. There is a considerable literature on the eigenvalues of doubly stochastic matrices, see for instance $[11,12,13,16,19,20]$. We review those results on this topic which we will use.

We begin with Fiedler's bound [11]. Let $M=\left(m_{i j}\right)$ be a doubly stochastic $N \times N$ matrix. Fiedler defined the index of irreducibility of $M$ to be

$$
\mu(M)=\min _{\mathcal{S}}\left\{\sum_{i \in \mathcal{S}, j \notin \mathcal{S}} m_{i j}\right\},
$$

where the minimum is over sets $\emptyset \subsetneq \mathcal{S} \subsetneq\{1, \ldots, N\}$. Note that $0 \leq \mu(M) \leq 1$, with $\mu(M)=0$ if and only if $M$ is reducible.

Lemma 4.1. [11, Theorem 3.4] Let $\lambda$ be any nonleading eigenvalue of the doubly stochastic $N \times N$ matrix $M$. Then $|1-\lambda| \geq 2(1-\cos (\pi / N)) \mu(M)$.

A related result of Fiedler and Ptak bounds the eigenvalues away from -1 in the case that $N$ is odd:

Lemma 4.2. [13, Theorem 3.4] Let $M$ be a doubly stochastic $N \times N$ matrix with $N$ odd. Then any eigenvalue $\lambda$ of $M$ satisfies $|1+\lambda| \geq(1-\cos (\pi / N)) \mu(M)$.

We next mention a result of Kellogg and Stephens which bounds the eigenvalues of a nonnegative matrix in terms of the associated directed graph. More precisely, given a nonnegative matrix $G=\left(g_{i j}\right)$ of order $N$, the directed graph $\mathcal{G}$ of $G$ has vertices $\{1, \cdots N\}$ and a directed edge $(i, j)$ if $g_{i j}>0$. A sequence $i_{1}, i_{2}, \cdots, i_{k}$ of distinct vertices is a closed circuit of $\mathcal{G}$ if $\mathcal{G}$ contains the edges $\left(i_{1}, i_{2}\right),\left(i_{2}, i_{3}\right), \ldots,\left(i_{k}, i_{1}\right)$. Therefore, the distinct vertices $i_{1}, i_{2}, \ldots, i_{k}$ form a circuit if and only if the product $g_{i_{1} i_{2}} \cdots g_{i_{k}, i_{1}} \neq 0$. The length of this circuit is $k$. 
Lemma 4.3. [19, Theorem 1] Let $G$ be a nonnegative matrix with directed graph $\mathcal{G}$ and spectral radius $\rho$. Let $\kappa$ be the length of the longest circuit of $\mathcal{G}$. If $\kappa=2$, all the eigenvalues of $G$ are real. If $\kappa>2$, each eigenvalue $\lambda$ of $G$ satisfies

$$
\operatorname{Re}(\lambda)+|\operatorname{Im}(\lambda)| \tan \left(\frac{\pi}{\kappa}\right) \leq \rho .
$$

We will also need the following standard result.

Lemma 4.4. (Weyl's inequalities) [5, Theorem III.2.1] Let $A, B$ be two real symmetric $N \times N$ matrices with eigenvalues $\alpha_{1} \geq \ldots \geq \alpha_{N}$ and $\beta_{1} \geq \ldots \geq \beta_{N}$. Let $\gamma_{1} \geq \ldots \geq \gamma_{N}$ be the eigenvectors of $A+B$. Then

$$
\gamma_{j} \leq \alpha_{i}+\beta_{j-i+1} \text { for } i \leq j, \quad \gamma_{j} \geq \alpha_{i}+\beta_{j-i+N} \text { for } i \geq j .
$$

4.2. The extremal property of $f_{\mathrm{zz}}$. In this subsection, we prove Theorem 2, using Theorem 3 and Fiedler's bound, Lemma 4.1. We first prove (1.1).

Fix $i$ and let $A=A\left(f, N_{i}\right)$. We consider the doubly stochastic matrix $m^{-2} A^{T} A$. As $\operatorname{gcd}\left(m, N_{i}\right)=1$ and $m$ is odd, it follows from Lemma 3.5 that this matrix is primitive, and hence irreducible. As its entries lie in $m^{-2} \mathbb{Z}$, we have $\mu\left(m^{-2} A^{T} A\right) \geq$ $m^{-2}$. Moreover, $m^{-2} A^{T} A$ is symmetric and positive semidefinite, so its eigenvalues are real and nonnegative. Thus, writing $\theta_{i}=\pi / 2 N_{i}$, Lemma 4.1 gives

$$
1-\tau\left(m^{-2} A^{T} A\right) \geq 2 m^{-2}\left(1-\cos \left(2 \theta_{i}\right)\right)=4 m^{-2} \sin ^{2} \theta_{i} .
$$

But $\tau\left(m^{-2} A^{T} A\right) \geq m^{-2} \tau_{\text {perm }}(A)^{2}$ by Lemma 2.7 . Hence

$$
\begin{aligned}
1-m^{-1} \tau_{\text {perm }}(A) & =\frac{1-m^{-2} \tau_{\text {perm }}(A)^{2}}{1+m^{-1} \tau_{\text {perm }}(A)} \\
& \geq \frac{1}{2}\left(1-\tau\left(m^{-2} A^{T} A\right)\right) \\
& \geq 2 m^{-2} \sin ^{2} \theta_{i} .
\end{aligned}
$$

From (2.7), we have either $\tau^{\left(N_{i}\right)}(f)=m^{-1} \tau_{\text {perm }}(A)$ or $\tau^{\left(N_{i}\right)}(f)=m^{-1}$. If $\tau^{\left(N_{i}\right)}(f)=m^{-1} \tau_{\text {perm }}(A)$, we therefore have

$$
1-\tau^{\left(N_{i}\right)}(f) \geq 2 m^{-2} \sin ^{2} \theta_{i} .
$$

As $m \geq 3$, this also holds if $\tau^{\left(N_{i}\right)}(f)=m^{-1}$.

On the other hand, from Theorem 3 we have

$$
1-\tau^{\left(N_{i}\right)}\left(f_{\mathrm{zz}}\right)=\frac{m \sin \theta_{i}-\sin \left(m \theta_{i}\right)}{m \sin \theta_{i}} .
$$

Thus

$$
\liminf _{i \rightarrow \infty} \frac{1-\tau^{\left(N_{i}\right)}(f)}{1-\tau^{\left(N_{i}\right)}\left(f_{\mathrm{zz}}\right)} \geq \lim _{\theta \rightarrow 0} \frac{2 m^{-1} \sin ^{3} \theta}{m \sin \theta-\sin (m \theta)}=\frac{12}{m^{4}-m^{2}} .
$$

This completes the proof of inequality (1.1).

We now turn to the proof of (1.2). Thus we take $m=3$ and suppose that $\operatorname{gcd}(3, N)=1$. By Lemma 2.8, there are only three maps $f \in \mathcal{F}_{3}$ to consider, namely $f_{\mathrm{sf}}, f_{\mathrm{zz}}$ and $f_{1,1,-1}$. By Theorems 3 and 4 , we have $\tau^{(N)}\left(f_{\mathrm{sf}}\right)<\tau^{(N)}\left(f_{\mathrm{zz}}\right)$, and it remains to show that $\tau^{(N)}(f) \leq \tau^{(N)}\left(f_{\mathrm{zz}}\right)$ for $f=f_{1,1,-1}$.

The $N \times N$ matrix $A(f, N)$ has constant row and column sums 3 , its entries being 0 or 1 apart from one occurrence of 2 in the final column. We define two related 
symmetric $\{0,1\}$ matrices $G=\left(g_{i j}\right)_{1 \leq i, j \leq N}$ and $P=\left(p_{i j}\right)_{1 \leq i, j \leq N}$ as follows:

$$
g_{i j}= \begin{cases}1 & \text { if } i+j=N-1 \text { or } N \\ 1 & \text { if }(i, j)=(N-1, N) \text { or }(N, N-1) \text { or }(N, N) ; \\ 0 & \text { otherwise; }\end{cases}
$$

and, writing $N=3 k+r$ with $r \in\{1,2\}$,

$$
p_{i j}= \begin{cases}1 & \text { if }\lceil i / 3\rceil+\lceil j / 3\rceil=k+1 \text { and } i+j \equiv r+1 \bmod 3, \\ 1 & \text { if } i=j>3 k ; \\ 0 & \text { otherwise. }\end{cases}
$$

Then the matrix $T=G+P$ is the unique symmetric matrix $T$ which can be obtained by permuting the rows of $A(f, N)$. To estimate $\tau^{(N)}(f)$, however, it is not sufficient to bound the eigenvalues of $T$ away from 1 using Lemma 4.1, since $T$ is not in general positive semidefinite. Instead, we use Weyl's inequalities (Lemma 4.4).

Let $\alpha_{1} \geq \ldots \geq \alpha_{N}$ and $\beta_{1} \geq \ldots \geq \beta_{N}$ be the eigenvalues of the nonnegative real symmetric matrices $G, P$ respectively. The rows of the anticirculant matrix $G$ can be permuted to give a symmetric circulant matrix $C$, in which each row has two consecutive entries 1 and all other entries 0 . We have $\tau(C)=2 \cos (\pi / N)$. (This follows from (3.2) when $N$ is odd, but is easily verified for all $N$.) Thus, by Lemma $2.7, \tau_{\text {perm }}(G) \leq 2 \cos (\pi / N)$. Hence $\alpha_{1}=2$ and $-2 \cos (\pi / N) \leq \alpha_{N} \leq \alpha_{2} \leq$ $2 \cos (\pi / N)$. On the other hand, $-1 \leq \beta_{N} \leq \ldots \leq \beta_{1} \leq 1$ since the only eigenvalues of the symmetric permutation matrix $P$ are \pm 1 . Now let $\lambda_{1}=3 \geq \lambda_{2} \geq \ldots \geq \lambda_{N}$ be the eigenvalues of $T$. For $j \geq 2$, Lemma 4.4 gives

$$
\lambda_{j} \leq \alpha_{2}+\beta_{j-1} \leq 2 \cos (\pi / N)+1 \text { and } \lambda_{j} \geq \alpha_{N}+\beta_{j} \geq-2 \cos (\pi / N)-1 .
$$

Hence

$$
\tau_{\text {perm }}(T) \leq 1+2 \cos (\pi / N)=3-4 \sin ^{2}(\pi / 2 N)
$$

so that

$$
\tau^{(N)}(f) \leq \frac{1}{3}\left(3-4 \sin ^{2}(\pi / 2 N)\right) .
$$

On the other hand, by Theorem 3 ,

$$
\tau^{(N)}\left(f_{\mathrm{zz}}\right)=\frac{\sin (3 \pi / 2 N)}{3 \sin (\pi / 2 N)}=\frac{1}{3}\left(3-4 \sin ^{2}(\pi / 2 N)\right) .
$$

So we have $\tau^{(N)}(f) \leq \tau^{(N)}\left(f_{\mathrm{ZZ}}\right)$, as required.

4.3. The tent map. In this subsection, we prove our final theorem, which concerns the tent map $f_{\text {tent }}$ for odd $N$.

Proof of Theorem 5. Let $m=2$ and let $N=2 s+1$. We first show that $\tau_{\text {mix }}^{(N)}\left(f_{\text {tent }}\right) \geq \tau^{(N)}\left(f_{\text {sf }}\right)$. From Theorem 4 , we have

$$
\tau^{(N)}\left(f_{\mathrm{sf}}\right)=\frac{\sin (2 \pi / N)}{2 \sin (\pi / N)}=\cos (\pi / N)
$$

Let $A=A\left(f_{\text {tent }}, N\right)=\left(a_{i j}\right)$; explicitly

$$
a_{i j}= \begin{cases}1 & \text { if } i=h \text { or } N+1-h \text { and } j=2 h-1 \text { or } 2 h \text { for } 1 \leq h \leq s \\ 2 & \text { if } i=s+1, j=N \\ 0 & \text { otherwise. }\end{cases}
$$


We can permute the rows of $A$ to get the matrix $D=\left(d_{i j}\right)$ such that

$$
d_{i j}= \begin{cases}1 & \text { if } i=1 \text { or } 3 \text { and } j=1 \text { or } 2 \\ 1 & \text { if } i=2 h-2 \text { or } 2 h+1 \text { and } j=2 h-1 \text { or } 2 h \text { for } 2 \leq h \leq s \\ 2 & \text { if } i=N-1, j=N \\ 0 & \text { otherwise. }\end{cases}
$$

Then $D$ is irreducible since the associated directed graph contains a circuit of length $N$ given by the sequence of edges

$$
(1,2),(2,4),(4,6), \cdots,(N-3, N-1),(N-1, N),(N, N-2), \cdots,(5,3),(3,1) .
$$

Thus $\tau_{\text {mix }}^{(N)}\left(f_{\text {tent }}\right) \geq \frac{1}{2} \tau(D)$.

The matrix $D$ has eigenvalue 0 with multiplicity $s$ and (leading) eigenvalue 2 with multiplicity 1 . We claim that the remaining $s$ nonleading eigenvalues are $\lambda_{r}=2 \cos (2 \pi r / N)$ for $1 \leq r \leq s$. We will then have $\tau_{\text {mix }}^{(N)}\left(f_{\text {tent }}\right) \geq \frac{1}{m} \tau(D)=$ $\frac{1}{2} \max _{r}\left|\lambda_{r}\right|=\cos (\pi / N)$, as required.

To prove the claim, we observe that a row vector of the form

$$
\left(\alpha_{s}, \alpha_{s}, \alpha_{s-1}, \alpha_{s-1}, \ldots, \alpha_{1}, \alpha_{1}, \alpha_{0}\right)
$$

is an eigenvector of $D$ with eigenvalue $\lambda$ if and only if

$$
\alpha_{s}+\alpha_{s-1}=\lambda \alpha_{s}, \quad \alpha_{h+1}+\alpha_{h-1}=\lambda \alpha_{h} \text { for } 1 \leq h \leq s-1, \quad 2 \alpha_{1}=\lambda_{0} .
$$

Fix $r$ with $1 \leq r \leq s$, let $\zeta=e^{2 \pi i r / N}$, and set $\alpha_{j}=\zeta^{j}+\zeta^{-j}$. Then the above equations hold with $\lambda=\lambda_{r}$; this follows easily on noting that $\alpha_{0}=2$, $\alpha_{h+1}+\alpha_{h-1}=\alpha_{1} \alpha_{h}$ and $\alpha_{s}=\alpha_{s+1}$. Thus $\lambda_{r}$ is indeed an eigenvalue of $D$, as claimed.

Next we prove (1.5) and (1.6). Let $\sigma \in S_{N}$ be a permutation such that $\sigma \circ f_{\text {tent }}$ is topologically mixing, and let $U_{\sigma}=\left(u_{i j}\right)=\frac{1}{2} A\left(\sigma \circ f_{\text {tent }}, N\right)$ be the corresponding $N \times N$ primitive, doubly stochastic matrix. Then $\mu\left(U_{\sigma}\right) \geq \frac{1}{2}$. Moreover, for each $\emptyset \subsetneq \mathcal{S} \subsetneq\{1, \cdots, N\}$, we have

$$
0<\sum_{i \in \mathcal{S}, j \notin \mathcal{S}} u_{i j}=\sum_{i \notin \mathcal{S}, j \in \mathcal{S}} u_{i j}=\sharp \mathcal{S}-\sum_{i \in \mathcal{S}, j \in \mathcal{S}} u_{i j} .
$$

Now let $V_{\sigma}=\frac{1}{2}\left(U_{\sigma}+U_{\sigma}^{T}\right)$. Then $V_{\sigma}$ is symmetric and doubly stochastic, and it follows from (4.1) that $\mu\left(V_{\sigma}\right)=\mu\left(U_{\sigma}\right)$. In particular, $V_{\sigma}$ is irreducible.

Let the eigenvalues of the real symmetric stochastic matrix $V_{\sigma}$ be $1=\eta_{1}>\eta_{2} \geq$ $\eta_{3} \geq \cdots \geq \eta_{N}>-1$. Let $\mathbf{y}$ be a unit eigenvector for a nonleading eigenvalue $\lambda$ of $\bar{U}_{\sigma}$. Then, writing $(\cdot, \cdot)$ for the usual complex inner product on $\mathbb{C}^{N}$, we have $\left(U_{\sigma} \mathbf{y}, \mathbf{y}\right)=\lambda(\mathbf{y}, \mathbf{y})$ and $\left(U_{\sigma}^{T} \mathbf{y}, \mathbf{y}\right)=\left(\mathbf{y}, U_{\sigma} \mathbf{y}\right)=\bar{\lambda}(\mathbf{y}, \mathbf{y})$. Hence,

$$
\operatorname{Re}(\lambda)=\left(V_{\sigma} \mathbf{y}, \mathbf{y}\right) \geq \eta_{N}
$$

Furthermore, since $N$ is odd, it follows from Lemma 4.2 that

$$
\operatorname{Re}(\lambda) \geq-1+\mu\left(V_{\sigma}\right)\left(1-\cos \left(\frac{\pi}{N}\right)\right) \geq-\cos \left(\frac{\pi}{2 N}\right)^{2},
$$

giving the first inequality of (1.5).

On the other hand, if $\lambda$ is a nonleading eigenvalue of $U_{\sigma}$ corresponding to the unit eigenvector $\mathbf{y}$, then

$$
\eta_{2}=\max _{\|\mathbf{x}\|=1, \mathbf{x} \in \mathbb{C}_{0}^{N}}\left(V_{\sigma} \mathbf{x}, \mathbf{x}\right) \geq\left(V_{\sigma} \mathbf{y}, \mathbf{y}\right)=\operatorname{Re}(\lambda) .
$$


Applying Fiedler's bound Lemma 4.1, we have

$$
1-\eta_{2} \geq 2\left(1-\cos \left(\frac{\pi}{N}\right)\right) \mu\left(V_{\sigma}\right) \geq 1-\cos \left(\frac{\pi}{N}\right),
$$

that is,

$$
\operatorname{Re}(\lambda) \leq \eta_{2} \leq \cos \left(\frac{\pi}{N}\right) .
$$

This completes the proof of (1.5).

Finally, we prove the inequality (1.6). Let $\kappa$ be the length of the longest circuit in the graph associated to $U_{\sigma}$. As $U_{\sigma}$ is irreducible, we have $\kappa>1$. Thus $2 \leq \kappa \leq N$, and, as $U_{\sigma}$ has spectral radius $\rho=1$, the Kellogg-Stephens bound Lemma 4.3 gives

$$
\operatorname{Re}(\lambda)+|\operatorname{Im}(\lambda)| \tan \left(\frac{\pi}{N}\right) \leq \operatorname{Re}(\lambda)+|\operatorname{Im}(\lambda)| \tan \left(\frac{\pi}{\kappa}\right) \leq 1
$$

as required.

The bound $\kappa=N$ in the last part of the preceding proof is attained by the matrix $D$ in the first part of the proof, since, as noted above, this matrix contains a circuit of length $N$.

\section{Appendix: Proofs of linear algebra Results}

We conclude by proving a few lemmas about linear algebra, which are used in $\S 2$ and $\S 3$.

5.1. Proof of Lemma 2.7. Let $A$ be a real $N \times N$ matrix with eigenvalues $\lambda_{1}, \ldots, \lambda_{N}$, numbered so that $\left|\lambda_{1}\right| \geq\left|\lambda_{2}\right| \geq \ldots \geq\left|\lambda_{N}\right|$. The singular values of $A$ are the real numbers $s_{1} \geq s_{2} \geq \ldots \geq s_{N} \geq 0$ such that $s_{1}^{2}, \ldots, s_{N}^{2}$ are the eigenvalues of each of the symmetric, positive semidefinite matrices $A A^{T}$ and $A^{T} A$ $[5, \S$ I.2]. For $1 \leq k \leq N$, using [5, (II.24)], we have

$$
\prod_{i=1}^{k}\left|\lambda_{i}\right| \leq \prod_{i=1}^{k} s_{i}
$$

Now let $A$ also be nonnegative and have constant row and column sums $m$. We may assume $m \neq 0$. Then $\lambda_{1}=s_{1}=m,\left|\lambda_{2}\right|=\tau(A)$ and $s_{2}^{2}=\tau\left(A A^{T}\right)=\tau\left(A^{T} A\right)$. By (5.1) with $k=2$, we have $m \tau(A) \leq m \sqrt{\tau\left(A^{T} A\right)}$, so that $\tau(A)^{2} \leq \tau\left(A A^{T}\right)$. Replacing $A$ by $A P(\sigma)$ for $\sigma \in S_{N}$, we have $\tau(A P(\sigma))^{2} \leq \tau\left(A P(\sigma) P(\sigma)^{T} A^{T}\right)=$ $\tau\left(A A^{T}\right)$. As this holds for all $\sigma$, it follows that $\tau_{\text {perm }}(A)^{2} \leq \tau\left(A A^{T}\right)$, giving the first assertion of Lemma 2.7.

Suppose further that $A$ is symmetric. Then the eigenvalues $\lambda_{j}$ are real and the corresponding eigenvalues $\mathbf{e}_{j}$ can be chosen to form an orthogonal basis of $\mathbb{C}^{N}$ with respect to the usual complex inner product $(\cdot, \cdot)$. Then

$$
\left(A^{T} A \mathbf{e}_{i}, \mathbf{e}_{j}\right)=\left(A \mathbf{e}_{i}, A \mathbf{e}_{j}\right)=\left(\lambda_{i} \mathbf{e}_{i}, \lambda_{j} \mathbf{e}_{j}\right)=\lambda_{i} \lambda_{j}\left(\mathbf{e}_{i}, \mathbf{e}_{j}\right)=\lambda_{i}^{2} \delta_{i j} .
$$

Thus the eigenvalues of $A^{T} A$ are the $\lambda_{j}^{2}$, so that $\tau\left(A^{T} A\right)=\lambda_{2}^{2}=\tau(A)^{2}$. As $\tau_{\text {perm }}(A) \geq \tau(A) \geq 0$ by definition, and we have shown $\tau(A)^{2}=\tau\left(A^{T} A\right) \geq$ $\tau_{\text {perm }}(A)^{2}$, it follows that $\tau_{\text {perm }}(A)=\tau(A)$. 
5.2. Collapsing block matrices. In this subsection, we prove Lemmas 2.4 and 3.2. To do so, we need to "collapse" certain $N \times N$ matrices (viewed as $n \times n$ matrices of $d \times d$ blocks, where $N=n d$ ), to obtain $n \times n$ matrices. For $1 \leq p \leq N$, we write $p=(i-1) d+r$ with $1 \leq i \leq n$ and $1 \leq r \leq d$. Thus a subscript $p$ relates to the $r$ th position in the $i$ th block. To emphasize this indexing by pairs $(i, r)$, we will write the entries $b_{p q}$ of an $N \times N$ matrix $B$ as $b_{i j}^{(r s)}$ in place of $b_{(i-1) d+r(j-1) d+s}$.

We will say that $B$ has the column block property for $d$ if the $d$ columns in each block are identical:

$$
b_{i j}^{(r s)}=b_{i j}^{(r 1)} \text { for } 1 \leq i, j \leq n \text { and } 1 \leq r, s \leq d .
$$

We then define $B^{\downarrow}=\left(b_{i j}^{\downarrow}\right)$ to be the $n \times n$ matrix obtained by replacing each block in $B$ by the sum of any of its columns:

$$
b_{i j}^{\downarrow}=\sum_{r=1}^{d} b_{i j}^{(r s)} .
$$

(This sum is independent of $s$ because of the column block property).

The next result is a slightly modified version of [7, Lemma 4.2].

Lemma 5.1. Let $B$ be an $N \times N$ matrix with constant row and column sums, and with the column block property for $d$. Then

$$
\tau\left(B^{\downarrow}\right)=\tau(B) .
$$

Proof. For $1 \leq j \leq n$ and $1 \leq s \leq d$, let $\mathbf{v}_{j}^{(s)} \in \mathbb{C}^{N}$ be the vector whose component in position $(i-1) d+r$ is as follows: if $s=1$ then

$$
v_{j i}^{(s r)}= \begin{cases}1 & \text { if } r=1, i=j, \\ 0 & \text { otherwise }\end{cases}
$$

and if $s \neq 1$ then

$$
v_{j i}^{(s r)}= \begin{cases}-1 & \text { if } r=1, i=j, \\ 1 & \text { if } r=s, i=j, \\ 0 & \text { otherwise. }\end{cases}
$$

As $B$ has the column block property, we have $B \mathbf{v}_{j}^{(s)}=\mathbf{0}$ if $s \neq 1$. Thus the linear endomorphism $\theta$ of $\mathbb{C}^{N}$ given by $B$ vanishes on the $n(d-1)$-dimensional subspace $W$ spanned by the $\mathbf{v}_{j}^{(s)}$ with $s \neq 1$. It follows that $\theta$ induces an endomorphism $\bar{\theta}$ of the quotient space $\mathbb{C}^{N} / W$. The $n$ cosets $\mathbf{v}_{j}^{(1)}+W$ form a basis for this quotient space, and the matrix for $\bar{\theta}$ with respect to this basis is given by replacing each $d \times d$ block in $B$ by the sum of one of its (identical) columns. This matrix is precisely $B^{\downarrow}$. The eigenvalues of $\theta$ are those of $\bar{\theta}$, with the same multiplicities, together with the eigenvalue 0 with a (further) multiplicity $n(d-1)$. Thus, if $n \geq 2$, we have $\tau\left(B^{\downarrow}\right)=\tau(B)$. In the degenerate case $n=1$, we have $\tau(B)=0$ since the only nonleading eigenvalue of $B$ is 0 (with multiplicity $d-1$ ), and by definition $\tau\left(B^{\downarrow}\right)=0$.

Proof of Lemma 2.4. Let $g=\sigma \circ f$ with $\sigma \in S_{N}$. Then the $N m \times N m$ matrix $B(f, N)$ has the column block property with $d=m$ by $(2.2)$, and hence so does $B(g, N)$ since this property is preserved under multiplying on the right by the block permutation matrix $Q(\sigma)$. Indeed, if we partition $B(g, N)$ into $m \times m$ blocks, and 
collapse each block to the sum of any one of its columns, the resulting $N \times N$ matrix $B(g, N) \downarrow$ is precisely $A(g, N)$, since each block of $m$ consecutive rows corresponds to a single interval of length $1 / N$. Thus $\tau(A(g, N))=\tau(B(g, N))$ by Lemma 5.1.

Proof of Lemma 3.2. Let $A$ be a nonnegative $d \times d$ matrix with constant row and columns sums, and let $B=A^{\uparrow}$ be the corresponding $N \times N$ matrix as in Definition 3.1. Then $B$ has the column block property, and $B^{\downarrow}=d A$. Thus, using Lemma 5.1, $\tau\left(A^{\uparrow}\right)=\tau(B)=\tau\left(B^{\downarrow}\right)=d \tau(A)$.

5.3. Proof of Proposition 3.4. Let $C=C(m, N)$ with $\operatorname{gcd}(m, N)=1$ be the symmetric circulant matrix defined in $\S 3.1 .3$. Let $\eta=e^{\pi i / N}$, let $\omega_{j}=\eta^{2 j}$ for all $j \in \mathbb{Z}$, and set

$$
\mathbf{v}_{j}=\left(1, \omega_{j}, \omega_{j}^{2}, \ldots, \omega_{j}^{N-1}\right)^{T} \in \mathbb{C}^{N} .
$$

A routine calculation (cf. [7, p. 3394]) then shows that $C \mathbf{v}_{j}=\lambda_{j} \mathbf{v}_{j}$ where

$$
\lambda_{j}= \begin{cases}m & \text { if } N \mid j, \\ (-1)^{(m-1) j} \frac{\sin (m j \pi / N)}{\sin (j \pi / N)} & \text { otherwise. }\end{cases}
$$

Now set $\mathbf{w}_{j}=\eta^{j} \mathbf{v}_{j}$. Then $J \mathbf{w}_{j}=\mathbf{w}_{-j}=\mathbf{w}_{2 N-j}=-\mathbf{w}_{N-j}$. For $1 \leq j<N / 2$, define

$$
\mathbf{f}_{j}=\mathbf{w}_{j}+\mathbf{w}_{-j}=\mathbf{w}_{j}-\mathbf{w}_{N-j} \text { and } \mathbf{g}_{j}=\mathbf{w}_{j}-\mathbf{w}_{-j}=\mathbf{w}_{j}+\mathbf{w}_{N-j} .
$$

These vectors, together with $\mathbf{w}_{0}$ and (in the case that $N$ is even) $\mathbf{w}_{N / 2}$ clearly form a basis of $\mathbb{C}^{N}$. We will show that they are all eigenvectors for $D=D(m, N)$. Certainly $D \mathbf{w}_{0}=2 m \mathbf{w}_{0}$, giving the leading eigenvalue $2 m$. Now we calculate

$$
\begin{aligned}
D \mathbf{f}_{j} & =\left(C \mathbf{w}_{j}+C J \mathbf{w}_{j}-C \mathbf{w}_{n-j}-C J \mathbf{w}_{n-j}\right) \\
& =\left(C \mathbf{w}_{j}-C \mathbf{w}_{n-j}-C \mathbf{w}_{n-j}+C \mathbf{w}_{j}\right) \\
& =2\left(\lambda_{j} \mathbf{w}_{j}-\lambda_{n-j} \mathbf{w}_{n-j}\right) \\
& =2 \lambda_{j} \mathbf{f}_{j} .
\end{aligned}
$$

Thus $\mathbf{f}_{j}$ is an eigenvector of $D$ with eigenvalue $2 \lambda_{j}$. Similar calculations show that $D \mathbf{g}_{j}=\mathbf{0}$ and (if $n$ is even) $D \mathbf{w}_{n / 2}=\mathbf{0}$. Hence the nonzero nonleading eigenvalues of $D$ are $2 \lambda_{j}$ for $1 \leq j<n / 2$. The result now follows from (3.2).

\section{ACKNOWLEDGMENTS}

The authors would thank the "Probability in Dynamics" workshop at IM-UFRJ, Rio de Janeiro, in 26-30 May, 2014. We also thank an anonymous referee for his/her very helpful comments. Y. Zhang is partially supported by FONDECYT, grant No. 3130622 .

\section{REFERENCES}

[1] P. Ashwin, M. Nicol and N. Kirkby, Acceleration of one-dimensional mixing by discontinuous mappings. Phys. A, 310, (2002), 347-363. 1

[2] R. Badii, K. Heinzelmann, P.F. Meier and A. Politi, Correlation functions and generalized Lyapunov exponents. Phys. Rev. A, 37 (1988), 1323-1328. 1

[3] V. Baladi, Positive Transfer Operators and Decay of Correlations. (Advanced Series in Nonlinear Dynamics 16), World Scientific Publishing Co. Inc., River Edge, NJ, 2000. 1 
[4] V. Baladi, S. Isola and B. Schmitt Transfer operator for piecewise affine approximations of interval maps, Ann. Inst. H. Poincare Phs. Théor. 62, 251-262, (1995). 1

[5] R. Bhatia, Matrix Analysis. (Graduate Texts in Mathematics 169), Springer, New York, 1997. 4.4, 5.1

[6] A. Boyarsky and P. Gora. Laws of Chaos. Invariant measures and dynamical systems in one dimension. (Probability and its Applications), Birkhauer, Boston, MA, 1997. 1

[7] N.P. Byott, M. Holland and Y. Zhang, On the mixing properties of piecewise expanding maps under composition with permutations. Discrete Contin. Dyn. Syst. 33, (2013), 3365-3390. 1, $1,2,2,3.1 .3,3.3,5.2,5.3$

[8] F. Christiansen, G. Paladin and H.H. Rugh, Determination of correlation spectra in chaotic systems. Phys. Rev. Lett. 65 (1990), 2087-2090. 1

[9] P. Collet and J.-P. Eckmann, Liapunov multipliers and decay of correlations in dynamical systems. J. Statist. Phys., 115, (2004), 217-254. 1

[10] M. Dellnitz, G. Froyland and S. Sertl, On the isolated spectrum of the Perron-Frobenius operator. Nonlinearity, 13, (2000), 1171-1188. 1

[11] M. Fiedler, Bounds for eigenvalues of doubly stochastic matrices. Linear Algebra Appl., 5, (1972), 299-310. 1, 4.1, 4.1

[12] M. Fiedler, A Minimaximin Formula and its Application to Doubly Stochastic Matrices. Mat. Časopis Sloven. Akad. Vied, 25(2), (1975), 139-144. 4.1

[13] M. Fiedler and V. Pták, A quantitative extension of the Perron-Forbenius theorem for doubly stochastic matrices. Czechoslovak Math. J., 25, (1975), 339-353. 4.1, 4.2

[14] G. Froyland. Finite approximation of Sinai-Bowen-Ruelle measures of Anosov systems in two dimensions, Random and Comp. Dyn. 3 64, 251-0264, (1995). 1

[15] G. Froyland. Computer-assisted bounds for the rate of decay of correlations, Commun. Math. Phys, 189, 237-257, (1997). 1

[16] C.R. Johnson. An inclusion region for the field of values of a doubly stochastic matrix based on its graph. Aequationes Math. 17, (1978), 305-310. 4.1

[17] W. Just, Analytical treatment of fluctuation spectra at the symmetry breaking chaos transition. Phys. Lett. A, 150, (1990), 362-368. 1

[18] G. Keller, On the Rate of convergence to Equilibrium in One-Dimensional Systems. Commun. Math. Phys., 96, (1984), 181-193. 2

[19] R.B. Kellogg and A.B. Stephens. Complex eigenvalues of a non-negative matrix with a specified graph. Linear Algebra and Appl., 20(2), (1978), 179-187. 4.1, 4.3

[20] U.G. Rothblum and C.P. Tan, Upper bounds on the Maximum modulus of subdominant eigenvalues of nonnegative matrices. Linear Algebra Appl., 66, (1985), 45-86. 4.1

[21] J. Slipantschuk, O. Bandtlow and W. Just, On the relation between Lyapunov exponents and exponential decay of correlation, J. Phys A., 46, (2013). 1

[22] J. Slipantschuk, O. Bandtlow and W. Just, On correlation decay in low-dimensional systems Europhys. Lett. EPL 10420004 (2013). 1

[23] S .M. Ulam. Problems in Modern Mathematics. New York, 1964. 1

[24] M. Viana. Stochastic Dynamics of Deterministic Systems. Braz. Math. Colloq. 21, IMPA, 1997. 1

(Nigel P. Byott) College of Engineering, Mathematics and Physical Sciences, University of EXETER, Exeter, UK.

E-mail address: N.P.Byott@exeter.ac.uk

(Congping Lin) College of Engineering, Mathematics and Physical Sciences, UNIVERSity of Exeter, EXeter, UK.

E-mail address: C.Lin@exeter.ac.uk

(Yiwei Zhang) Facultad de Matemáticas, Pontificia Universidad Católica de Chile, Avenida Vicuña Mackenna 4860, Santiago, Chile

E-mail address: yiweizhang831129@gmail.com 